\title{
Research on MOOC Educational Platform Model based on Satellite Communication Technology
}

\author{
Yi Shang, Bao-ming Li, Guang-qiang Yang \\ Space Star Technology Co., Ltd ,Beijing, China
}

\begin{abstract}
Currently MOOC models of distance education based on internet are widely used in the international education fields, but MOOC education model mainly cover fields with developed internet environment. In the poor internet environment, MOOC education model can not effectively be carried out. So a MOOC education platform based on satellite communications are provided, which enables internet-restricted area quickly covered by MOOC educational resources and provides an effective complement to the promotion of education MOOC.
\end{abstract}

Keywords - satellite communication, MOOC, distance education

\section{基于卫星通信技术的 MOOC 教育平台模式的研究}

尚壹 李宝明杨广强

航天恒星科技有限公司, 北京, 中国

摘 要 当前基于互联网的 MOOC 方式的远程教育在国际教育领域被广泛采用, 但 MOOC 教育模式覆盖区域主要在互联网较为 发达地区, 在互连网欠发达区域 MOOC 教育模式不能有效开展, 本文提出的基于卫星通信方式的 MOOC 教育平台能够实现互联网受 限区域 MOOC 教育资源的快速覆盖, 为 MOOC 教育的推广提供有效补充。

关键词 卫星通信, MOOC, 远程教育

\section{1. 引言}

远程教育模式随着时代进步在不断变化, 由最初的传 统函授教育模式, 到无线电波式广播教育模式, 再到电视 教育模式以及后来的网络课程教育模式, 直到最近基于互 联网+理念的 MOOC 模式。

MOOC 即大型开放式网络课程, 是远程教育领域近期 广泛研究发展的结果。 ${ }^{[1]} \mathrm{MOOC}$ 教学方式近年来发展迅速, 已成为远程教学领域的佼佼者, 甚至 2012 年在教育界被称 为 MOOC 年。MOOC 教学特点是以 “短视频+交互式练习” 为基本教学单元的知识点/知识体组织模式和学习模式, 有 助于学生的 “碎片化” 学习。 ${ }^{[2]} \mathrm{MOOC}$ 未来可预见发展的 趋势包括 MOOC 规模将进一步扩大、关联 MOOC 将会成 为未来慕课的发展方向, 教师教育理念与方法将产生巨变, 学生的学习方法将大为改观、网络技术将推动教育的巨大 变革、现行教育体制将深受冲击。 ${ }^{[3]}$ 由此可见, MOOC 已 掀起了远程教育领域的一次革命, 使更多的人享受的优秀 的教育资源, MOOC 将在线学习、社交服务、大数据分析、
移动互联网等理念融为一体, 其特点是师生之间, 同学之 间频繁的互动, 因此目前 MOOC 覆盖区域主要是互联网较 为发达地区, 而仍有许多地区由于缺乏互联网接入或者互 联网带宽资源有限而失去通过 MOOC 学习的机会, MOOC 的教育资源对于这些互联网受限的区域尤为宝贵。因此解 决 MOOC 对互联网接入受限区域的覆盖和连通问题是实 现 MOOC 广泛传播真正意义的关键所在。本文就如何能够 快速实现 MOOC 教育资源对互联网接入受限区域的覆盖 进行了分析研究, 并提出了基于卫星通信技术的 MOOC 教 育平台方案。

\section{2. 卫星通信技术}

目前卫星通信技术发展迅猛, 其不受地理环境和距离 的约束, 点到多点、点到面的覆盖优势和灵活的可移动性, 是任何其它通信手段都无法相比的, 因此卫星通信技术被 的广泛的应用于远程教育领域, 并取得了很好的效果。卫 星通信技术主要分为广播和双向通信两种方式, 其中基于 
广播方式的卫星应用技术包括电视广播技术、数据广播技 术以及 PushVOD 技术等, 基于双向通信的卫星应用技术主 要是 VSAT 通信技术。

\section{1 卫星广播技术}

\section{a. 电视广播技术}

属于单向卫星通信技术, 电视信号经上行站将信号发 送到通信卫星, 通过通信卫星信号转发器向卫星信号波束 覆盖范围的地面区域转发, 用户通过卫星电视接收终端 (接 收天线+LNB+机顶盒）即可获得电视信号, 以此技术为基 础的卫星电视教育频道目前仍在广泛使用。

\section{b. 数据广播技术}

数据广播的技术使用单一的系统前端(Headend), 连 续、滚动地将已编辑整理好的内容, 经过卫星传输网络传 输到用户终端, 用户通过具备接收数据广播功能的机顶盒 完成内容接收和解码, 其特点是广播内容不是单一的视频 资源, 而是由视频、音频、软件程序、流式数据或者其他 数字多媒体组成的多媒体资源, 用户在本地可以通过遥控 器在电视上选择需要观看的内容。数据广播技术通过轮播 方式使用户在单向广播环境下可以具有交互式通信的体 验。

\section{c. PushVOD 技术}

PushVOD 技术主要是解决卫星资源的问题, 节目在一 个集中的时段内通过卫星广播网络推送出去, PushVOD 终 端机顶盒需可以在该时段内将节目内容下载到本地, 终端 有一个存储的介质 (现在普遍使用硬盘介质), 用户实际使 用的时候, 系统只需要进行终端的本地操作即可 ${ }^{[4]}$ 。通常 PushVOD 推送资源只占用闲时卫星网络资源, 实现了为用 户提供个性化大容量的服务目的, 推送内容是由视频、音 频、软件程序、流式数据或者其他数字多媒体组成的多媒 体资源。

\section{2 卫星双向通信技术}

VSAT 通信技术即甚小口径卫星终端站技术, 属于卫 星双向通信技术, 是卫星固定通信业务的主要实现方式, VSAT 通信系统由空间和地面两部分组成, 地面部分由主 站和端站组成, 空间部分主要是通信卫星。

\section{3. 基于卫星通信技术的 $\mathrm{MOOC}$ 平台}

不同区域互联网资源大体可分为 4 个等级, 分别对应 (1 级) 好、(2 级) 一般、(3 级) 差、(4 级) 无。 1 级 2 级互联网区域通常具备宽带接入环境, 如 VDSL, ADSL 等; 3 级区域通常网络带宽不超过 100kbps, 如电话拨号方 式访问, 4 级区域没有互联网接入环境。考虑卫星通信及
MOOC 的特点, 可建立几种基于不同卫星技术的 MOOC 教育平台, 以覆盖 3 级和 4 级具备不同互联网资源环境区 域。

\section{1 基于卫星双向通信技术的 $\mathrm{MOOC}$ 平台}

当前卫星主流通信频段由 $\mathrm{C} 、 \mathrm{Ku}$ 逐步向 $\mathrm{Ka}$ 转移, 数 据传输率越来越大, $\mathrm{Ku}$ 及 $\mathrm{Ka}$ 频段 VSAT 系统可以提供不 同品质的宽带互联网接入服务[5]。因此根据卫星通信频段 划分大体可分为两类 $\mathrm{MOOC}$ 平台, 一类是基于 $\mathrm{C} 、 \mathrm{Ku}$ 频 段信号的常规 VSAT-MOOC 系统, 一类是基于 Ka 频段信 号的 Ka 信关站-MOOC 系统。

a. 常规 VSAT-MOOC 系统

1) 组成

常规 VSAT-MOOC 平台是基于 $\mathrm{C}$ 和 $\mathrm{Ku}$ 频段 VSAT 通 信技术的 MOOC 平台, 使用 $\mathrm{Ku}$ 或 $\mathrm{C}$ 频段通信卫星, 通过 透明转发, 采用 DVB-S2 链路传输协议和 IP 网络协议完成 信息传输, 实现信号波束覆盖区域内远程终端的网络接入, 接入数据率最高可达 $10 \mathrm{Mbps}$ 左右。主要包括 $\mathrm{Ku} / \mathrm{C}$ VSAT HUB、 $\mathrm{Ku} / \mathrm{C}$ 频段通信卫星、MOOC 服务平台、远程终端 四大部分。用户通过远程终端访问 MOOC 服务平台, 采用 非对称音视频交互方式完成教育和学习过程。

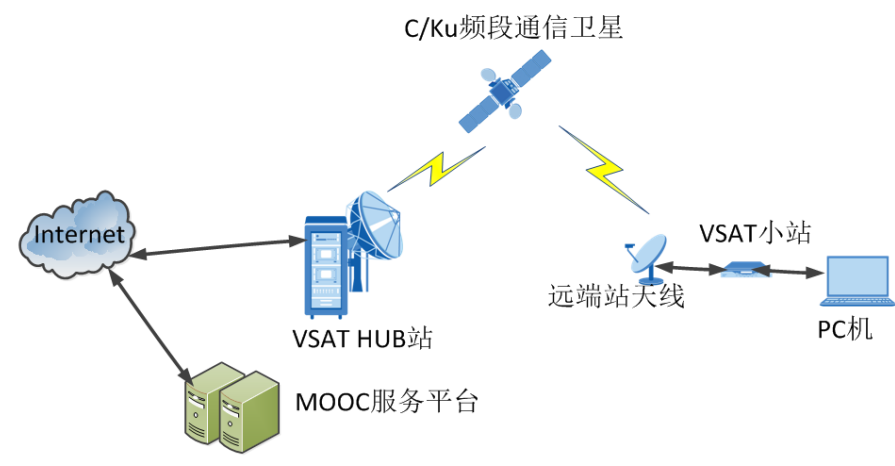

图 1 常规 VSAT-MOOC 系统结构图

2) 功能及特点

$>$ 系统功能包括课程管理、课程资源管理、交流互动、 卫星网络管理、学习管理

支持互联网访问;

$>$ 远程教育终端既可以是老师端, 也可以是学生端。

$>$ 提供课程直播、点播功能;

$>$ 能够支持对一般视频课程的正常访问;

$>$ 能够提供文本、语音及一般分辨率视频的交流互动;

$>$ 能够提供 $\mathrm{QQ}$ 、微信等即时通讯工具交流;

$>$ 终端能够快速扩展部署。 
b. Ka 信关站-MOOC 系统

1) 组成

$\mathrm{Ka}$ 信关站技术是一种具有大容量, 高速率数据传输的 VSAT 通信技术 ${ }^{[5]}$, 通常使用 Ka 频段多点波束静止轨道区 域覆盖通信卫星, 通过透明转发, 采用 DVB-S2 扩展链路 传输协议和 IP 网络协议完成信息传输, 实现波束覆盖区域 面积内远程终端最高可达 $100 \mathrm{Mbps}$ 数据率的通信吞吐量。 主要包括 $\mathrm{Ka}$ 信关站、 $\mathrm{Ka}$ 通信卫星、MOOC 服务平台、远 程接入终端四大部分。信关站各主站之间通过地面网络互 联, 远程接入终端通过对应 $\mathrm{Ka}$ 信关站访问 MOOC 服务平 台。

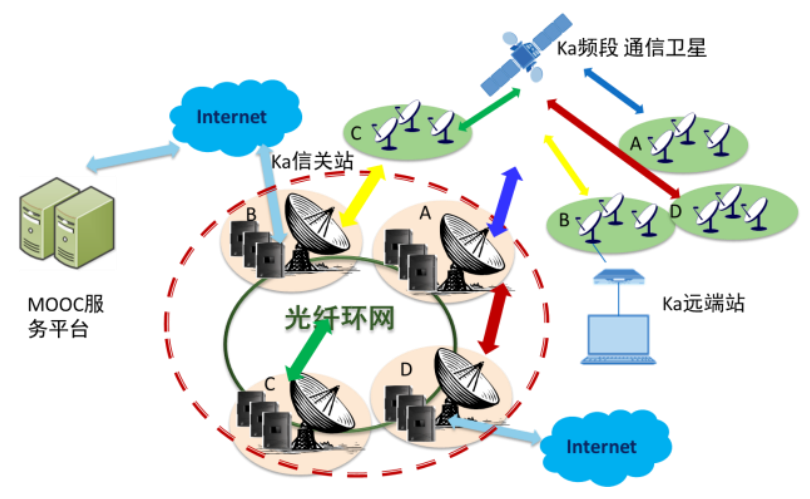

图 $2 \mathrm{Ka}$ 信关站-MOOC 系统结构图

\section{2）功能及特点}

系统功能包括课程管理、课程资源管理、交流互动、 卫星网络管理、学习管理;

支持互联网访问, 能够提供非常高的网络访问速率; 远程教育终端既可以是老师端、也可以是学生端。

提供课程直播、点播功能;

能够支持对高清视频课程的正常访问;

能够提供文本、语音及高分辨率视频的互动交流; 能够快速扩展部署;

\section{2 基于卫星广播技术的 $\mathrm{MOOC}$ 平台}

结合有限互联网带宽回传技术和卫星电视广播技术、 数据广播技术实现建立面向卫星广播服务的 MOOC 平台。

\section{a. 组成}

主要包括卫星电视上行站、MOOC 服务平台、数据广 播系统、电视终端、手机或连接有限带宽互联网的 PC 机 终端, MOOC 平台按照预先安排好的数据广播节目表通过 卫星电视信号广播课程数据, 同时学员可通过手机短信、 移动互联网或者连接有限带宽网络 (如电话拨号访问方式) 的 PC 机与 $\mathrm{MOOC}$ 平台的其他用户进行语音及文本互动。

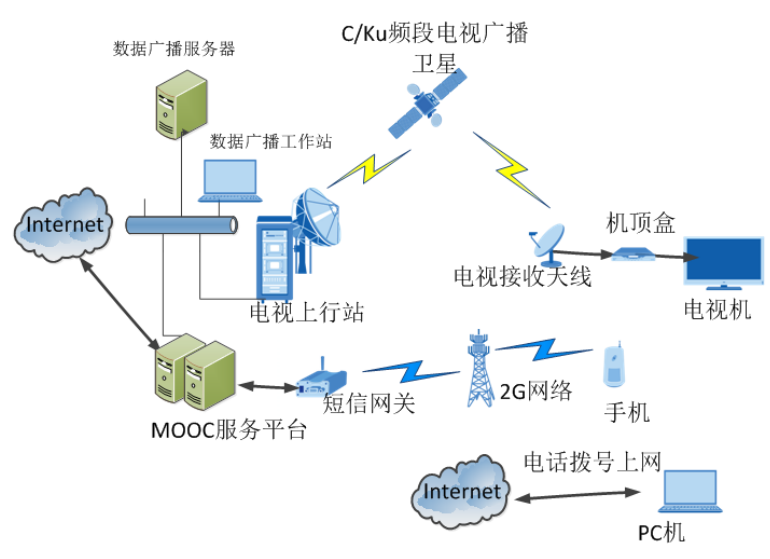

图 3 基于卫星广播技术的 MOOC 系统结构图

b. 功能及特点

$>$ 服务平台功能包括课程管理、课程资源管理、交流互 动、学习管理。

$>$ 系统提供数据广播信号输出;

$>$ 电视终端、手机或者连接有限带宽互联网的 PC 机终 端主要作为学生端使用;

$>$ 支持有限带宽的移动互联网 (如 $2 \mathrm{G}$ 网络) 交互;

$>$ 支持有限带宽 (网络带宽小于 100kbps) 的地面互联 网交互, 如电话拨号网络 (网络带宽通常在 64kbps 左 右)。

$>$ 能够为广播电视用户快速提供 $\mathrm{MOOC}$ 平台资源共享;

$>$ 能够通过手机短信进行有限互动交流;

$>$ 教师端仍需要具有较好的互联网接入环境。

\section{3 应用分析}

通常大规模建设地面网络的投入要远大于通信卫星建 设费用, 且耗时较长。因此, 通过建设或者租赁通信卫星 资源, 卫星通信网络能够能够快速完成 MOOC 教育资源接 入，与地面网络建设方式而言，投资小见效快。

在没有互联网资源的 4 级区域, 可以根据实际情况采用卫 星通信技术方式实现 MOOC 教育资源接入。如果利用现有 卫星资源, 由于 $\mathrm{Ku}$ 频段和 $\mathrm{C}$ 频段通信卫星资源较多, 因 此常规 VSAT-MOOC 系统建设更加容易, Ka 信关站-MOOC 系统由于受 $\mathrm{Ka}$ 频段资源卫星分布限制, 只能在有条件的地 区才能建立实施。但 $\mathrm{Ka}$ 频段是未来卫星通信的发展趋势, 随着未来 $\mathrm{Ka}$ 资源卫星资源数量不断增长, $\mathrm{Ka}$ 信关站 -MOOC 系统将会得到广泛应用。

在互连网资源受限的 3 级区域, 可以根据当地实际情 况, 运用单向的卫星广播技术实现 MOOC 资源的接入, 通 过手机短信及有限带宽的互联网连接实现该区域用户对 MOOC 资源的使用。 


\section{4. 结论}

本文通过对 MOOC 应用宗旨和卫星通信技术特点的 研究, 提出了基于卫星通信技术的 MOOC 教育平台模式, 该平台模式能够有效解决互联网资源贫乏地区 MOOC 资 源的接入和使用问题, 实现 MOOC 资源对偏远地区用户的 覆盖。

\section{参考文献(References)}

[1] Bozkurt, A., Akgun-Ozbek, E., Onrat-Yilmazer, S., Erdogdu, E., Ucar, H., Guler, E., Sezgin, S., Karadeniz, A., Sen, N., Goksel-Canbek, N., Dincer, G. D., Ari, S.,\& Aydin, C. H. (2015). Trends in Distance Education Research: A Content Analysis of
Journals 2009-2013. International Review of Research in Open and Distributed Learning, 16(1), 330-363.

[2] Sun Maosong, Look at MOOC at view of technology and research, China Computer Federation Communication, 2013, 9(10), Tsinghua University.

[3] Guo Yingjian, Six trends of MOOC, China education network, http://webplus.cqmu.edu.cn/s/123/t/616/a/52515/info.jspy, 2014-3-24.

[4] Hu Xiaodong ,PushVOD discuss based on digital TV, Cable TV Technology,2009,(9)49-51.

[5] Joseph Merchlinsky etc., Ground Segment Technologies for Ka-Band and Beyond, 2013,(10). 